\section{Can LV Dyssynchrony as Assessed with Phase Analysis on Gated Myocardial Perfusion SPECT Preferably Predict Response to CRT?}

TO THE EDITOR: I read with great interest the study by Henneman et al. (1) suggesting that response to cardiac resynchronization therapy (CRT) is related to the presence of left ventricular (LV) dyssynchrony as assessed by phase analysis with gated myocardial perfusion SPECT. The study confirmed that responders and nonresponders had comparable baseline characteristics, except for histogram bandwidth, which was significantly larger in responders than in nonresponders. The methods and interpretation of the results, however, raise several concerns:

It is well known that normal myocardial motion is complex, with 3 separate components: radial contraction, longitudinal shortening, and rotation. Myocardium has a transmural heterogeneity of contraction under normal conditions: Contractility decreases from subendocardium to subepicardium, and during a reduction of myocardial perfusion capable of inducing myocardial ischemia, the subendocardial layer undergoes contractility impairment even in the absence of functional impairment of the subepicardial layer (2,3). A study by Helm et al. (4) confirmed that dyssynchrony as assessed by longitudinal motion could be of a sensitivity different from that of dyssynchrony as assessed by circumferential motion, follows a time course different from that of dyssynchrony as assessed by circumferential motion, and may manifest a benefit from CRT during specific cardiac phases depending on pacing mode. In a study by Henneman et al. (1), however, a total of 90 projections was obtained over a $360^{\circ}$ circular orbit and data were reconstructed by filtered backprojection and then reoriented to yield gated short-axis images. That is to say, LV dyssynchrony was assessed from circumferential motion. Are the sensitivity and specificity for the prediction of response to CRT by gated myocardial perfusion SPECT better if LV dyssynchrony is assessed from longitudinal motion? Are the sensitivity and specificity for the prediction of response to CRT by gated myocardial perfusion SPECT better if LV dyssynchrony is assessed from both longitudinal motion and circumferential motion?

\section{REFERENCES}

1. Henneman MM, Chen J, Dibbets-Schneider P, et al. Can dyssynchrony as assessed with phase analysis on gated myocardial perfusion SPECT predict response to CRT? J Nucl Med. 2007;48:1104-1111.

2. Song ZZ, Ma J. Analysis of myocardial deformation based on ultrasonic pixel tracking to determine transmurality in chronic myocardial infarction. Eur Heart J. 2007;28:1173-1174

3. Lima JAC, Jeremy R, Guier W, et al. Accurate systolic wall thickening by nuclear magnetic resonance imaging with tissue tagging: correlation with sonomicrometers in normal and ischemic myocardium. J Am Coll Cardiol. 1993;21:1741-1751.

4. Helm RH, Leclercq C, Faris OP, et al. Cardiac dyssynchrony analysis using circumferential versus longitudinal strain: implications for assessing cardiac resynchronization. Circulation. 2005;111:2760-2767.

Ze-Zhou Song

Zhejiang University

Hangzhou, China

COPYRIGHT (C) 2008 by the Society of Nuclear Medicine, Inc.

DOI: 10.2967/jnumed.107.049601
REPLY: We appreciate the important comments regarding our recent study on left ventricular (LV) dyssynchrony assessment with gated myocardial perfusion SPECT and its application in the prediction of response to cardiac resynchronization therapy (CRT) (1). We agree with Dr. Song that myocardial contraction is a complex 3-dimensional process. In our study, all patients underwent gated myocardial perfusion SPECT before CRT implantation, and data were reconstructed by filtered backprojection and then reoriented to yield gated short-axis images. Accordingly, Dr. Song suggests that in our study, LV dyssynchrony was assessed from circumferential motion. However, this is not entirely correct.

Phase analysis uses a count-based method to extract amplitude and phase from the regional LV count changes throughout the cardiac cycle. These count changes are a function of the change in thickness in the radial, circumferential, and longitudinal directions. From the stacked LV short-axis datasets, 3-dimensional count distributions are extracted and are subsequently submitted to Fourier analysis. Phase analysis uses a first-harmonic fast Fourier transform to extract 3-dimensional regional phases (phase arrays). These calculated phase arrays describe the regional onset of mechanical contraction of the myocardium in 3 dimensions (2).

Dr. Song questions whether sensitivity and specificity for prediction of response to CRT may be better when LV dyssynchrony is assessed from longitudinal motion or from both longitudinal and circumferential motion. Because phase analysis with gated myocardial perfusion SPECT, as applied in our study, takes into account myocardial contraction in all 3 dimensions, we cannot provide this information. This count-based method has at least 2 advantages over wall-motion-based methods: First, because it does not need a frame of reference to assess wall motion, it is more objective; second, because it directly assesses myocardial contraction, it is much less affected by passive wall motion, which can take place in a noncontracting region that is tethered rather than actively contracting. We believe that gated myocardial perfusion SPECT phase analysis, by including all 3 components of myocardial contraction, is an attractive alternative for LV dyssynchrony assessment.

\section{REFERENCES}

1. Henneman MM, Chen J, Dibbets-Schneider P, et al. Can LV dyssynchrony as assessed with phase analysis on gated myocardial perfusion SPECT predict response to CRT? J Nucl Med. 2007;48:1104-1111.

2. Chen J, Garcia EV, Folks RD, et al. Onset of left ventricular mechanical contraction as determined by phase analysis of ECG-gated myocardial perfusion SPECT imaging: development of a diagnostic tool for assessment of cardiac mechanical dyssynchrony. J Nucl Cardiol. 2005;12:687-695.

Maureen M. Henneman
Jeroen J. Bax
Leiden University Medical Center
Leiden, The Netherlands
Ji Chen
Ernest V. Garcia
Atlanta, Georgia

DOI: 10.2967/jnumed.107.049700 